\title{
Sequential treatment of HPV E6 and E7-expressing TC-1 cells with bortezomib and celecoxib promotes apoptosis through p-p38 MAPK-mediated downregulation of cyclin D1 and CDK2
}

\author{
JEE-EUN KIM ${ }^{1,2^{*}}$, JI-IN LEE $^{1 *}$, DONG-HOON JIN ${ }^{2}$, WANG JAE LEE ${ }^{3}$, GA BIN PARK $^{4}$, \\ SEONGHAN KIM ${ }^{4}$, YEONG SEOK KIM ${ }^{4}$, T.-C. WU ${ }^{5-8}$, DAE YOUNG HUR ${ }^{4}$ and DAEJIN KIM ${ }^{4}$
}

\begin{abstract}
${ }^{1}$ Department of Anatomy, Chung-Ang University, College of Medicine, Seoul; ${ }^{2}$ Institute for Innovate Cancer Research, Asan Medical Center, University of Ulsan College of Medicine, Seoul; ${ }^{3}$ Department of Anatomy, Seoul National University, College of Medicine, Seoul; ${ }^{4}$ Department of Anatomy and Laboratory for Cancer Immunotherapy, Inje University, College of Medicine, Busan, Republic of Korea; Departments of ${ }^{5}$ Pathology, ${ }^{6}$ Obstetrics and Gynecology, ${ }^{7}$ Molecular Microbiology and Immunology, and ${ }^{8}$ Oncology, Johns Hopkins Medical Institutions, Baltimore, MD, USA
\end{abstract}

Received January 2, 2014; Accepted February 27, 2014

DOI: $10.3892 / o r .2014 .3082$

\begin{abstract}
Interruption of the cell cycle is accompanied by changes in several related molecules that result in the activation of apoptosis. The present study was performed to verify the apoptotic effects of sequential treatment with bortezomib and celecoxib in TC-1 cells expressing the human papillomavirus (HPV) E6 and E7 proteins. In TC-1 cells sequentially treated with bortezomib and celecoxib, apoptosis was induced through decreased expression of signal transducer and activator of transcription-3 (STAT3), cyclin D1 and cyclin-dependent kinase (CDK) 2, which are major regulators of the G0/G1 cell cycle checkpoint. In addition, increased levels of p21, CHOP, $\mathrm{BiP}$ and p-p38 MAPK were identified in these cells. The treatment-induced apoptosis was effectively inhibited by treatment with SB203580, an inhibitor of p-p38. Moreover, the growth of tumors sequentially treated with bortezomib and celecoxib was retarded compared to the growth of tumors exposed to a single treatment with either bortezomib or celecoxib in vivo. We demonstrated that sequential treatment with bortezomib and celecoxib induced apoptosis via p-p38-mediated G0/ G1 cell cycle arrest and endoplasmic reticulum (ER) stress. Sequential treatment with these two drugs could therefore be a useful therapy for cervical cancer.
\end{abstract}

Correspondence to: Professor Dae Young Hur or Dr Daejin Kim, Department of Anatomy, Inje University, College of Medicine, Bokji-ro 75, Busanjin-Gu, Busan 614-735, Republic of Korea

E-mail: dyhur@inje.ac.kr

E-mail: kimdj@inje.ac.kr

*Contributed equally

Key words: bortezomib, celecoxib, TC-1, p38 MAPK, cyclin D1

\section{Introduction}

Human papillomavirus (HPV) infection is a major cause of cervical cancer, one of the most common cancers in women worldwide $(1,2)$. HPV DNA integration into the host genome results in constitutive expression of the viral oncoproteins E6 and E7, which deregulate cell cycle control through interactions with the tumor-suppressor protein $\mathrm{p} 53$ and $\mathrm{pRb}$-type oncoproteins (3). The HPV E6 protein induces the degradation of p53, thereby inhibiting p53-dependent signaling and contributing to tumorigenesis (4). The HPV E7 protein binds to the retinoblastoma family of proteins (pRb, p107 and p130) and prevents G1 arrest in response to a variety of antiproliferative signals (5).

$\mathrm{pRb}$ is important for progression of the cell cycle from the G1 to the S phase, which is regulated by the cyclin-dependent kinases (CDKs) cyclin D-CDK4/6 (6) and cyclin E-CDK2 (7). Cyclin and CDK inhibitors, such as $\mathrm{p} 16^{\mathrm{INK} 4 \mathrm{~A}}$ and $\mathrm{p} 21^{\mathrm{CIP} 1}$, regulate CDK activity. $\mathrm{p} 21^{\mathrm{CIP1}}$ activation by $\mathrm{p} 53$ inhibits the formation of cyclin E-CDK 2 complexes and triggers cell cycle arrest in the G1 phase (8). Therefore, many tumors exhibit increased cyclin D1 or CDK4 levels, and a loss of p16 ${ }^{\mathrm{INK} 4 \mathrm{~A}}$, $\mathrm{Rb}$ and $\mathrm{p} 53$. A previous study demonstrated that the cell cycle regulator proteins cyclin D1 and cyclin E1 are overproduced in cervical cancer cells (9). When TC-1 cells, which are immortalized mouse lung epithelial cells, were transduced with HPV E6 and E7, p53 and Rb expression in the TC-1 cells was reduced as a result of degradation mediated by interaction with HPV16 E6 and E7 (10). Combining bortezomib treatment with DNA vaccines was found to result in improved immunity against TC-1 cells in mice compared to monotherapy, and treatment with bortezomib increased TC-1 tumor cell apoptosis (11). However, the present study did not identify the mechanism of apoptosis since cyclin-dependent kinase inhibitors (CKIs), such as p21, are transcriptionally activated primarily by $\mathrm{p} 53$ during the G1 phase.

Bortezomib reversibly inhibits the $26 \mathrm{~S}$ proteasome, preventing the degradation of pro-apoptotic proteins related to 
the regulation of cell proliferation and survival. Many previous studies have shown that bortezomib decreases $N F-\kappa B$ and STAT3 activation as well as activation of their downstream target genes, ICAM-1, MCP-1 and cyclin D1 $(12,13)$. For these reasons, many clinical trials are in progress to test the effect of bortezomib alone or in combination with chemo/radiation to treat various $\mathrm{NF}-\kappa \mathrm{B}$-dependent solid tumor models. Cyclooxygenase-2 (COX-2) is known to play an important role in carcinogenesis and cancer progression (14). Several studies have shown that COX-2 is overexpressed in cervical intraepithelial neoplasia, but not in normal cervical tissue $(15,16)$. The HPV16 E6 and E7 oncoproteins contribute to carcinogenesis by increasing COX-2 transcription by activating the EGFRRas-MAP kinase pathway (17). COX-2 and Ki-67 expression levels were found to be downregulated and neoangiogenesis was inhibited in cervical cancer patients treated with celecoxib (400 mg) twice daily (18). Moreover, celecoxib induced apoptosis in various cancer cell lines via a COX-2-independent mechanism, which may involve inhibition of $\mathrm{NF}-\kappa \mathrm{B}$ and STAT3 phosphorylation (19). From these results, we predicted that celecoxib, a specific inhibitor of COX-2, may have an important role in cancer treatment in various malignancies when combined with bortezomib.

We, therefore, investigated whether bortezomib treatment acts synergistically with celecoxib to increase therapeutic efficacy against TC-1 cells, in an HPV16 E6- and E7-expressing cervical cancer model. We assessed various combinations of bortezomib and celecoxib in TC-1 cells to determine the most effective cancer therapy with drugs in vitro and in vivo.

\section{Materials and methods}

Cells, reagents and animals. Bortezomib (provided by Janssen Korea) was prepared as a $7.8 \mathrm{mM}$ stock in PBS and stored at $-20^{\circ}$ C. Celecoxib (Toronto Research Chemicals Inc., Toronto, Ontario, Canada) was prepared as a $200 \mathrm{mM}$ stock in DMSO and stored at $-20^{\circ} \mathrm{C}$. Antibodies to STAT3, p21, cyclin D1, CHOP and $\beta$-actin, and horseradish peroxidase (HRP)conjugated goat anti-rabbit/mouse IgG were purchased from Santa Cruz Biotechnology (Santa Cruz, CA, USA). Antibodies to CDK2, CDK4, survivin, p38, p-p38, Bid, Bcl-2, Bax and caspase-3, -8 and -9 were purchased from Cell Signaling Technology (Beverly, MA, USA). An antibody to COX-2 was purchased from Cayman Chemical Co. (Ann Arbor, MI, USA). An antibody to BiP was purchased from Abcam (Cambridge, UK). SB203580 was purchased from Calbiochem (Darmstadt, Germany). C57BL/6 mice were acquired from the Chung-Ang Laboratory Animal Service (Seoul, Korea). All procedures were performed according to the approved protocols and in accordance with the recommendations of the Ethics Committee of the College of Medicine, Inje University and Chung-Ang University for the proper use and care of laboratory animals.

Cell culture conditions and cell viability assay. The TC-1 cells were cultured in RPMI-1640 medium (HyClone, Logan, UT, USA) supplemented with $10 \%$ fetal bovine serum (FBS; HyClone), $1 \%$ penicillin-streptomycin, $1 \%$ glutamine, $1 \%$ MEM-non essential amino acids, and $1 \%$ sodium pyruvate (Life Technologies, Grand Island, NY, USA) in a humidified incubator supplied with $5 \% \mathrm{CO}_{2}$ at $37^{\circ} \mathrm{C}(10)$. TC-1 cells were seeded into 96 -well plates at a density of $5 \times 10^{3}$ cells/well. The cells were pre-treated with bortezomib for $6 \mathrm{~h}$ and then treated with celecoxib for an additional $18 \mathrm{~h}$. For comparison, TC-1 cells were treated either with bortezomib or celecoxib alone, or co-treated for $24 \mathrm{~h}$. Finally, TC-1 cells pre-exposed to celecoxib for $6 \mathrm{~h}$ were then treated with bortezomib for $18 \mathrm{~h}$. Cell viability was determined by an MTT [3-(4,5-dimethylthiazol-2-yl)-2,5-diphenyltetrazolium bromide] assay using the Vybrant MTT cell assay kit (Molecular Probes, Eugene, OR, USA). The analysis was performed according to the manufacturer's instructions. The absorbance at $560 \mathrm{~nm}$ was measured using a microplate reader (Molecular Probes).

Western blot analysis for apoptosis-related proteins and cell cycle regulators. TC-1 cells were seeded into 24-well plates at a density of $3 \times 10^{4}$ cells/well. The TC- 1 cells were then treated with bortezomib and celecoxib for the indicated times. After the cells were pelleted and resuspended in lysis buffer, $40 \mu \mathrm{g}$ of total cell protein per sample was subjected to 8-15\% sodium dodecyl sulfate-polyacrylamide gel electrophoresis (SDS-PAGE). The proteins were then transferred to an Immobilon polyvinylidene difluoride membrane (Millipore, Bedford, MA, USA) for immunoblotting. The primary antibodies used were: anti-Bip, anti-CDK2, anti-CDK4, antiCHOP, anti-CyclinD1, anti-cleaved caspase-3, anti-caspase-8, anti-caspase-9, anti-p38, anti-p53, anti-p21, anti-Stat3 (1:1,000); and anti-Cox-2, anti-Bid, anti-Bax, anti-Bcl-2 and anti- $\beta$ actin $(1: 2,000)$. The primary antibodies were detected using horseradish peroxidase-conjugated goat anti-rabbit $(1: 2,000)$ or goat anti-mouse $(1: 10,000)$ secondary antibodies. Western blot analysis was performed using standard techniques, and the bands were visualized with the ECL system (Amersham Pharmacia Biotech, UK).

Flow cytometric analysis of apoptosis and the cell cycle. TC-1 cells were treated with bortezomib and celecoxib singly, in combination, and sequentially for $12 \mathrm{~h}$. The cells were then analyzed using an Annexin V-FITC detection kit (BD Pharmingen, San Diego, CA, USA) according to the manufacturer's instructions. The TC-1 cells were harvested and fixed in cold $70 \%$ ethanol at $4^{\circ} \mathrm{C}$ overnight. The next day, the cells were stained with a $50 \mu \mathrm{g} / \mathrm{ml}$ propidium iodide (PI) solution (Sigma, St. Louis, MO, USA) and $500 \mu 110 \mu \mathrm{g} / \mathrm{ml}$ RNase A (Sigma) for $30 \mathrm{~min}$ at $37^{\circ} \mathrm{C}$ in the dark. The cells were analyzed immediately using a FACSCalibur flow cytometer (Becton-Dickinson, San Diego, CA, USA).

In vivo tumor treatment using bortezomib and celecoxib. C57BL/6 mice (5-6 per group) were challenged with $5 \times 10^{4}$ TC-1 tumor cells by subcutaneous (s.c.) injection for the in vivo tumor treatment experiment. By day 8 , the average tumor size was $\sim 90-100 \mathrm{~mm}^{3}$. The mice were then divided into the following groups: control (no treatment), single treatment, co-treatment, and sequential treatment with bortezomib $(0.5 \mathrm{mg} / \mathrm{kg})$ and celecoxib $(5 \mathrm{mg} / \mathrm{kg})$. Drug treatment was administered via intraperitoneal (i.p.) injection. The sequential treatment group was administered bortezomib (day 8, 10, 12 and 14) followed by celecoxib (day 9, 11, 12 and 14) or celecoxib (day 8, 10, 12 and 14) followed by bortezomib (day $9,11,12$ and 14) daily. The mice in the co-treatment groups 
A

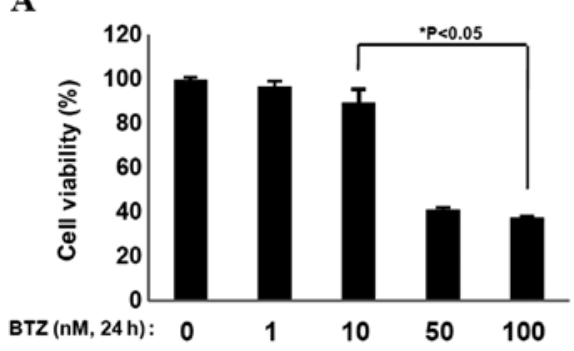

B

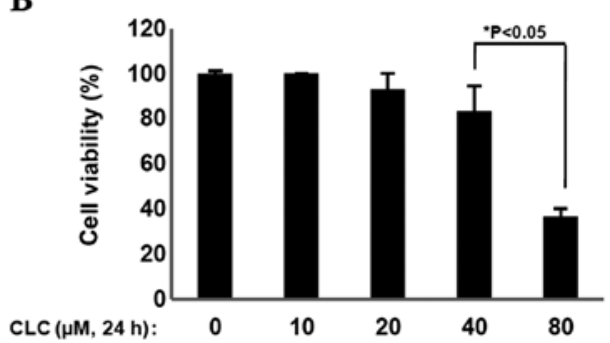

C

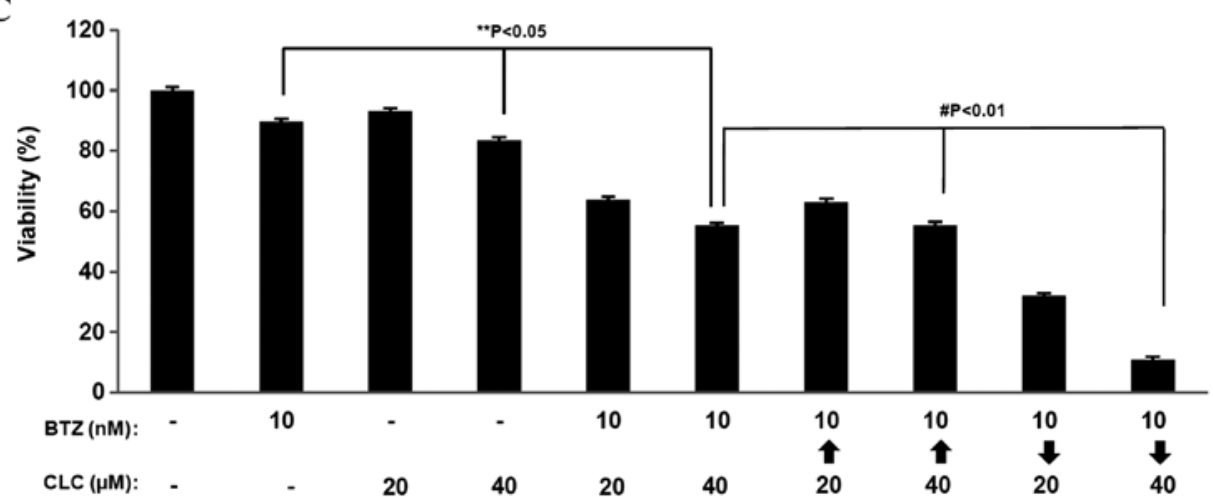

Figure 1. Effect of bortezomib and celecoxib on TC-1 cell viability. Survival of TC-1 cells was determined by MTT assay after 24 h of culture in the presence of increasing concentrations of bortezomib (BTZ) (A) and celecoxib (CLC) (B) ( $\mathrm{P}<0.05$; low dose vs. high dose). (C) Viability of TC-1 cells sequentially treated with bortezomib and celecoxib was assessed at the indicated concentrations. The cells were pre-incubated with bortezomib for $6 \mathrm{~h}$ and then treated with celecoxib for $18 \mathrm{~h}\left({ }^{* * *} \mathrm{P}<0.05\right.$, difference between bortezomib or celecoxib treatment and co-treatment; ${ }^{\#} \mathrm{P}<0.01$, difference between co-treatment or $\mathrm{CLC} \rightarrow \mathrm{BTZ}$ and $\mathrm{BTZ} \rightarrow \mathrm{CLC}$ ).

were injected with the appropriate drug four times every other day (day 8, 10, 12 and 14). The tumor size was measured at intervals of approximately two days. At the end of the treatment, the mice were sacrificed, and the tumors were collected and weighed.

Statistics. The mean value for cell viability or mean fluorescence intensity for the apoptosis assay with standard error of the mean (SEM) from representative experiments is shown in the figures. Data were confirmed by at least three independent experiments. All data shown are representative of at least two different experiments. The data were compared by analysis of variance (one-way ANOVA). Statistical significance was defined by a P-value of $<0.05$.

\section{Results}

Sequential treatment with bortezomib and celecoxib increases apoptosis via an intrinsic pathway in TC-1 cells. Treatment with bortezomib (BTZ) or celecoxib (CLC) significantly reduced cell viability at relative high doses $(50-100 \mathrm{nM}$ of bortezomib and $80 \mu \mathrm{M}$ of celecoxib) based on MTT assays $(\mathrm{P}<0.05)$ (Fig. 1A and B). However, co-treatment with relatively low doses of BTZ $(10 \mathrm{nM})$ and CLC (20 and $40 \mu \mathrm{M})$ induced significant levels of cell death in the MTT assays compared to single treatment $(\mathrm{P}<0.05$, individual drug treatment vs. co-treatment) (Fig. 1C). Notably, the effects of combination treatment with BTZ $(10 \mathrm{nM})$ and CLC $(40 \mu \mathrm{M})$ on cell viability showed strong differences depending on the treatment order $(\mathrm{P}<0.01$, sequential drug treatment vs. co-treatment). When TC-1 cells were treated with bortezomib and then celecoxib (BTZ $\rightarrow$ CLC), cell viability was significantly reduced compared to the group treated simultaneously with BTZ and CLC or the group treated with celecoxib and then bortezomib (CLC $\rightarrow$ BTZ) $(\mathrm{P}<0.01$, co-treatment or CLC $\rightarrow$ BTZ treatment vs. BTZ $\rightarrow$ CLC treatment) (Fig. 1C). The number of Annexin V-positive TC-1 cells increased after sequential treatment with bortezomib and celecoxib $(\mathrm{P}<0.001$, BTZ or CLC treatment vs. BTZ $\rightarrow$ CLC treatment) (Fig. 2A). Furthermore, production of pro-apoptotic protein Bax increased, whereas production of the anti-apoptotic protein Bcl-2 decreased after pre-treatment with bortezomib followed by treatment with celecoxib. Bid and caspase- 8 levels, which are related to the extrinsic apoptotic pathway, did not change after bortezomib and celecoxib treatment, while levels of cleaved caspase-3 and -9 increased in the TC- 1 cells that were sequentially treated with bortezomib and celecoxib (Fig. 2B and C). These results suggest that sequential treatment with relatively low doses of bortezomib and celecoxib may effectively induce TC-1 cell death through intrinsic apoptosis.

The apoptosis of TC-1 cells sequentially treated with bortezomib and celecoxib is mediated by STAT3, cyclin D1, CDK2 and ER stress. Co-treatment with bortezomib and celecoxib has been reported to induce apoptosis via production of the ER stress-related proteins, glucose-regulated protein 78 (GRP78/ $\mathrm{BiP})$ and CCAAT/enhancer binding protein homologous transcription factor (CHOP/GADD153), but not through inhibition of COX-2 production (20). Our results are consistent with these earlier studies, including the presence of increased COX-2 
A

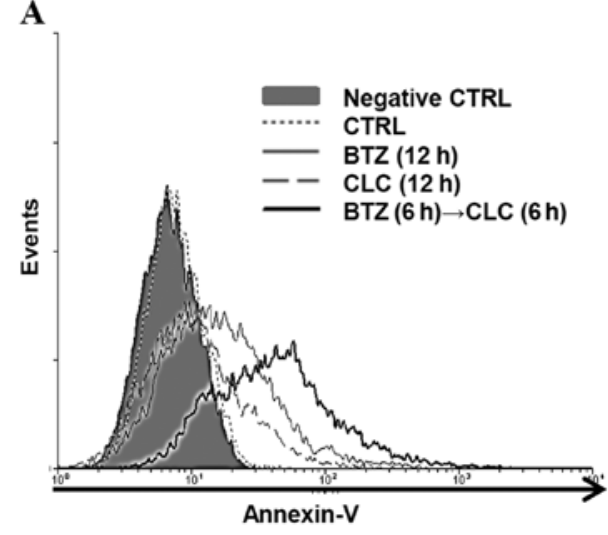

B

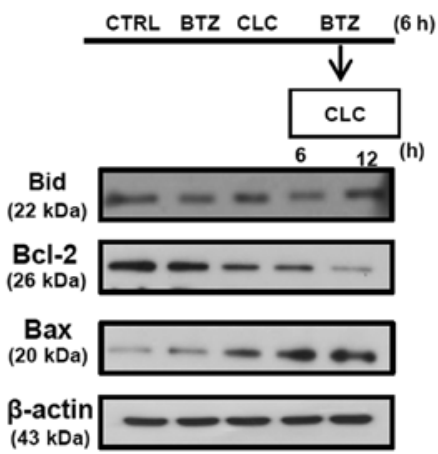

C
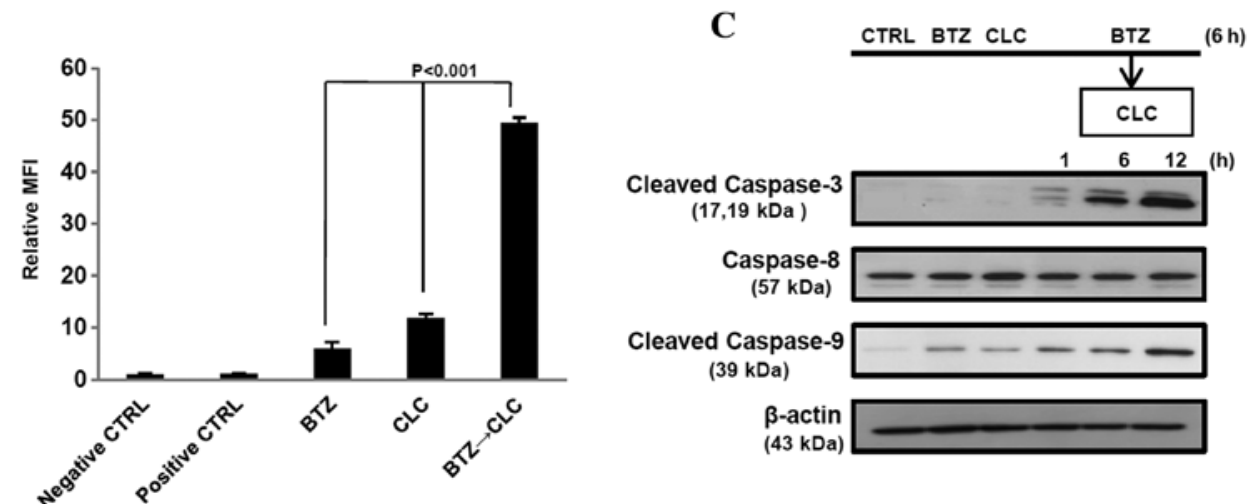

Figure 2. Effects of sequential bortezomib and celecoxib treatment on the induction of apoptosis and the expression of apoptosis-related proteins in TC-1 cells (A) The apoptosis rate after incubation with a single drug or combined sequential treatment with bortezomib $(10 \mathrm{nM})$ and celecoxib $(40 \mu \mathrm{M})$ was measured by Annexin V staining and flow cytometric analysis. The mean fluorescence intensity (MFI) of the apoptosis rate is also shown ( $\mathrm{P}<0.001$, difference between individual drug treatments and sequential bortezomib). (B) Expression of apoptosis-related Bcl-2 family proteins and (C) cleaved caspase-3 or -9 and caspase- 8 proteins was detected by western blotting. BTZ, bortezomib; CLC, celecoxib.

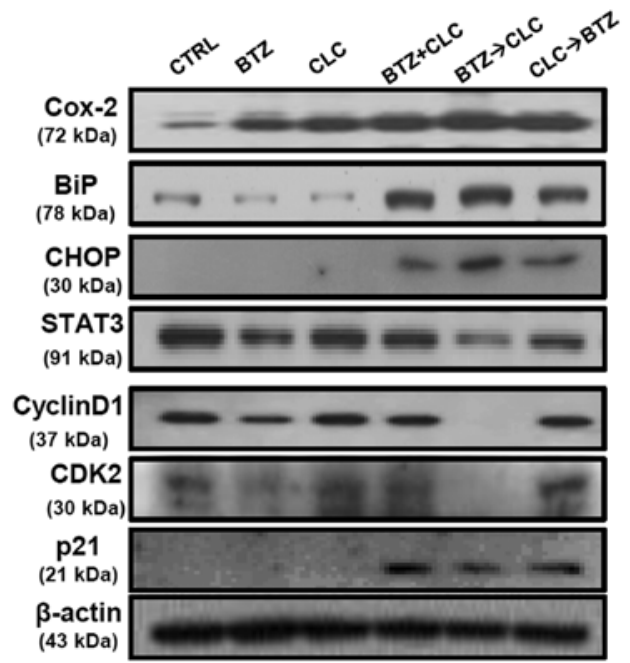

Figure 3. Mechanisms underlying the synergistic effect of sequential bortezomib and celecoxib treatment on apoptosis. Lysates were prepared from TC-1 cells treated with one or both drugs (BTZ, $10 \mathrm{nM}$; CLC, $40 \mu \mathrm{M}$ ) and immunoblotted using antibodies against ER stress-related proteins (BiP and CHOP) and G0/G1 cell cycle-related proteins (STAT3, cyclin D1, CDK2 and p21). BTZ, bortezomib; CLC, celecoxib.

levels after combined treatment with bortezomib and celecoxib (Fig. 3). However, the significant production of BiP and
CHOP is not sufficient to explain the high levels of apoptosis in TC-1 cells since TC- 1 cells co-treated with bortezomib and celecoxib or sequential celecoxib and bortezomib treatment also exhibited elevated $\mathrm{BiP}$ and CHOP protein levels (Fig. 3). STAT3 and cyclin D1 expression was significantly decreased in the TC-1 cells sequentially treated with bortezomib and celecoxib compared to the levels in the TC-1 cells co-treated with bortezomib and celecoxib or treated with a single drug. p21 production was not significantly upregulated in the TC-1 cells after single treatment with bortezomib or celecoxib. Co-treatment with bortezomib and celecoxib or sequential celecoxib and bortezomib treatment also enhanced $\mathrm{p} 21$ production in the p53-degraded TC-1 cells, while the levels of the cell cycle regulator proteins cyclin D1 and CDK2 did not change at all (Fig. 3). Notably, we observed that in the TC-1 cells sequentially treated with bortezomib and celecoxib, p21 production increased in a time-dependent manner and was associated with the downregulation of STAT3, cyclin D1, and CDK2, but not CDK4. BiP and CHOP levels were also significantly increased in the TC-1 cells sequentially treated with bortezomib and celecoxib in a time-dependent manner (Fig. 4A). In addition, we found that apoptosis in TC-1 cells sequentially treated with bortezomib and celecoxib was associated with cell cycle arrest in the $\mathrm{G} 0 / \mathrm{G} 1$ phase, and the apoptosis rate increased in a timedependent manner (Fig. 4B). These results suggest that the apoptosis of TC-1 cells sequentially treated with bortezomib 

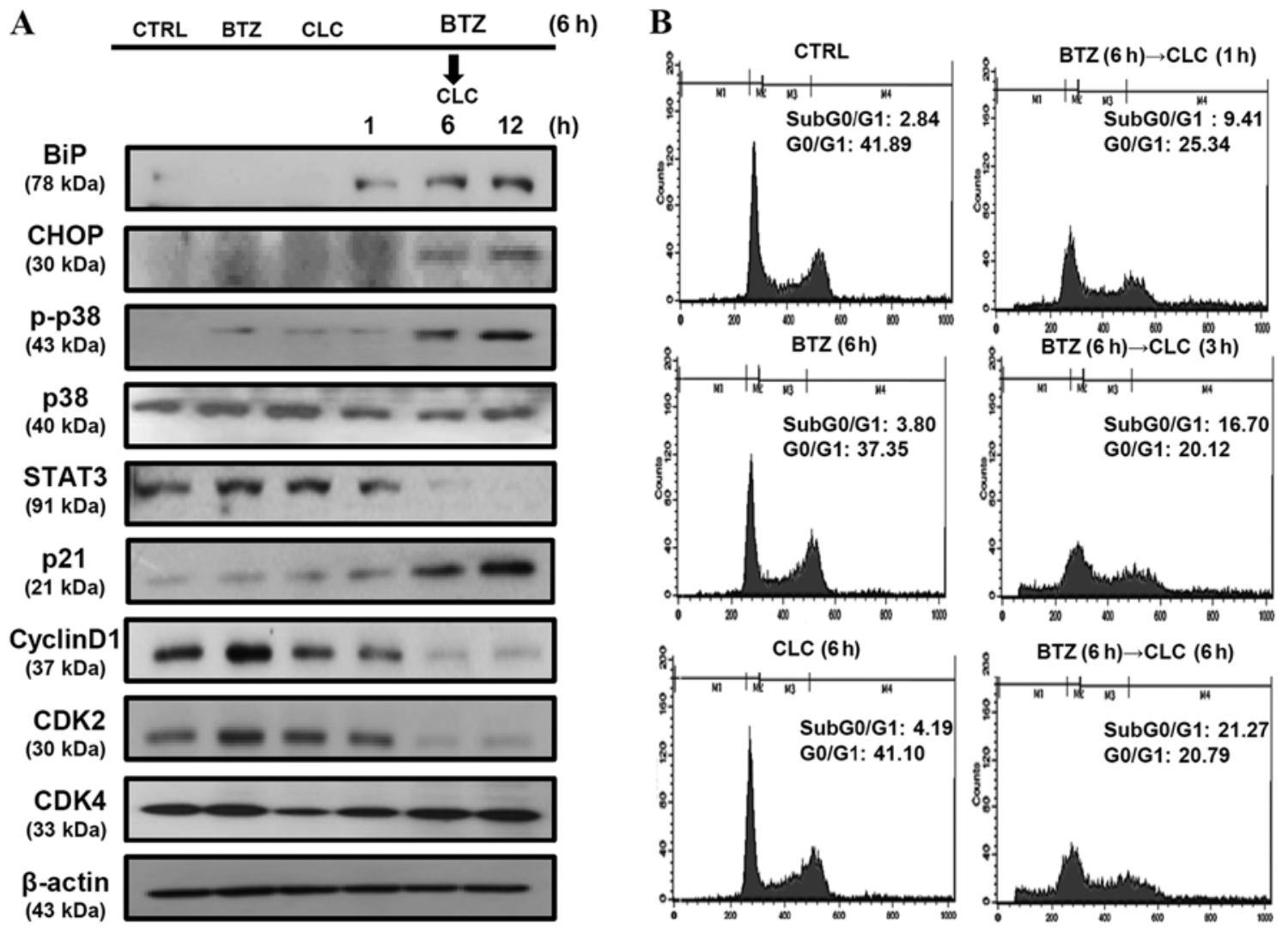

Figure 4. Sequential bortezomib and celecoxib treatment induces ER stress and cell cycle arrest in TC-1 cells. (A) Changes in expression of ER stress-related proteins (BiP and CHOP) and G0/G1 cell cycle-related proteins (p38 MAPK, p21, STAT3, cyclin D1, CDK2 and CDK4) after sequential treatment with bortezomib $(10 \mathrm{nM})$ and celecoxib $(40 \mu \mathrm{M})$ in TC-1 cells. (B) After incubation with combined sequential treatment, the cell cycle profile was analyzed by PI staining and FACS analysis. BTZ, bortezomib; CLC, celecoxib.

and celecoxib may be related to cell cycle regulation as well as ER stress.

Sequential treatment with bortezomib and celecoxib enhances TC-1 cell apoptosis via activation of p38 MAPK and inhibition of the cell cycle. p-p38 MAPK activation has been reported to mediate cell cycle arrest via an increase in p21 and p27 production and a decrease in the production of G1-specific cyclins (cyclin A, D1 and D3) and CDK4 and CDK6. Moreover, activation of p-p38 MAPK is associated with caspase activation as well as ER stress-induced apoptosis (21). We found that sequential treatment with bortezomib and celecoxib increased the expression of BiP, CHOP, p21 and phosphorylated p38 MAPK, but not unphosphorylated p38 MAPK, in a time-dependent manner in TC-1 cells (Fig. 4A). Next, we investigated the effects of activated p-p38 MAPK on BiP, CHOP, p21, STAT3, cyclin D1 and CDK2 using SB203580, an inhibitor of p-p38, in the TC-1 cells sequentially treated with bortezomib and celecoxib. When p-p38 was inhibited in these cells, production of STAT3, cyclin D1, CDK2 and antiapoptotic protein Bcl-2 was restored. In contrast, p21, Bax, cleaved caspase-3 and -9 were downregulated after treatment with SB203580 in these cells. However, SB203580 did not have an effect on BiP and CHOP production in the TC-1 cells sequentially treated with bortezomib and celecoxib (Fig. 5A and $\mathrm{B}$ ). The rate of apoptosis was also significantly decreased when p-p38 MAPK was inhibited by treatment with SB203580 $(\mathrm{P}<0.001, \mathrm{BTZ} \rightarrow \mathrm{CLC}$ treatment vs. BTZ $\rightarrow \mathrm{CLC}$ treatment after exposure to SB203580) (Fig. 5C). These results suggest that sequential treatment with bortezomib and celecoxib may induce apoptosis in TC-1 cells through a p-p38-mediated increase in p21 expression and cell cycle arrest, rather than through ER stress.

Sequential treatment with bortezomib and celecoxib retards tumor growth in TC-1 cell-inoculated mice. Finally, we determined whether sequential treatment with bortezomib and celecoxib inhibits the growth of TC-1 cells in vivo. C57BL/6 mice were challenged with an s.c. injection of TC-1 cells (5x10 $/$ mouse) 1 week prior to treatment with bortezomib or celecoxib or sequential treatment (Fig. 6A). We observed that the growth of TC-1 tumors sequentially treated with bortezomib and celecoxib was retarded compared to tumors co-treated with bortezomib and celecoxib or sequentially treated with celecoxib and bortezomib $(\mathrm{P}<0.05$, co-treatment or $\mathrm{CLC} \rightarrow \mathrm{BTZ}$ treatment vs. BTZ $\rightarrow \mathrm{CLC}$ treatment) (Fig. $6 \mathrm{~B}$ and $C$ ). Therefore, our data suggest that sequential treatment with bortezomib and celecoxib has greater therapeutic antitumor effects than each single treatment alone or any other bortezomib and celecoxib combination.

\section{Discussion}

The proteasome inhibitor bortezomib is currently used to treat various malignancies $(11,22)$, and the selective COX-2 inhibitor celecoxib has been used widely for chemoprevention (23). 
A$$
\text { A }
$$
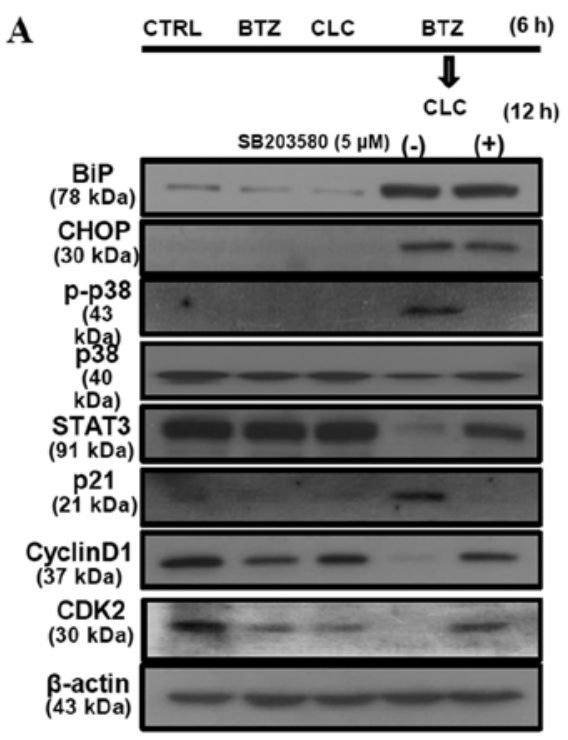

B
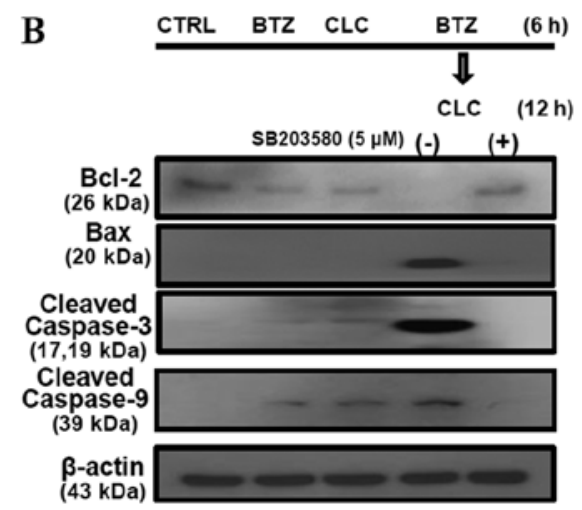
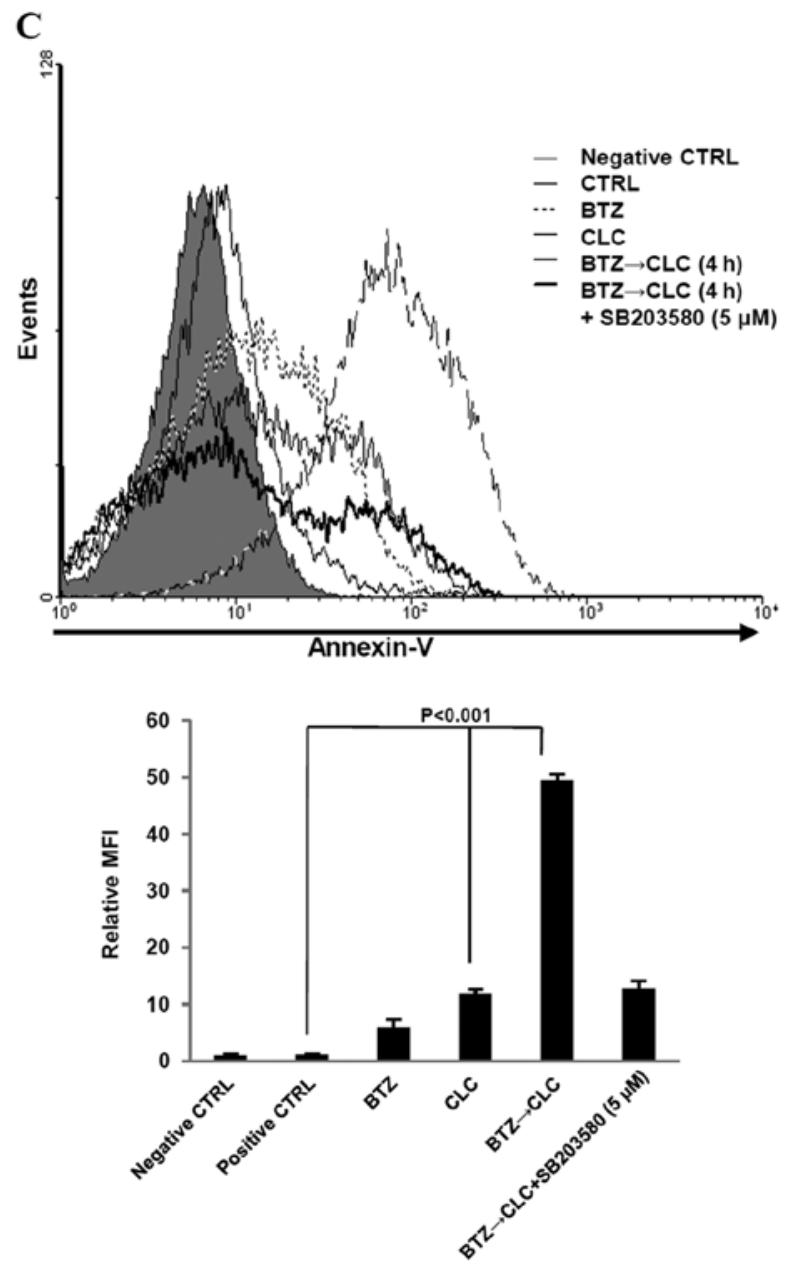

Figure 5. Effect of sequential bortezomib and celecoxib treatment on the induction of apoptosis via p-p38 MAPK-mediated cell cycle arrest in TC-1 cells. (A) The effect of SB203580 $(5 \mu \mathrm{M})$, a p-p38 MAPK inhibitor, on the expression of ER stress-mediated proteins and G0/G1 cell cycle-related proteins, as determined by western blotting. (B) The effect of SB203580, a p-p38 MAPK inhibitor, on the expression of apoptosis-related Bcl-2 family proteins and the caspase cascade was detected by western blotting. (C) The reduced rate of apoptosis induced by SB203580 before sequential treatment with bortezomib and celecoxib was measured by Annexin V (A-V) staining and flow cytometric analysis. The mean fluorescence intensity (MFI) of the apoptosis rate is also shown. $(\mathrm{P}<0.001$, difference between sequential bortezomib and celecoxib treatments and sequential bortezomib and celecoxib treatments after exposure to SB203580). BTZ, bortezomib; CLC, celecoxib.

In the present study, we demonstrated that pre-treatment with bortezomib followed by treatment with celecoxib enhanced apoptotic cell death through $\mathrm{p} 38$ MAPK-dependent dysregulation of cell cycle-related proteins including cyclin D1, CDK2 and p21, as well as ER stress in the HPV E6 and E7 proteinexpressing TC-1 cervical cancer model. The growth of tumors sequentially treated with bortezomib and celecoxib was retarded compared to tumors exposed to a single treatment with bortezomib or celecoxib in mice challenged with TC-1 cells.

Recent studies have shown that induction of apoptotic death by bortezomib is triggered via a c-Jun NH2-terminal kinase (JNK)-dependent mechanism and the accumulation of unfolded or misfolded proteins, which results in ER stress $(24,25)$. Bortezomib is thought to induce cell death by stimulating the expression of CHOP and other pro-apoptotic components (26). Celecoxib has shown potent anticancer activity in various animal tumor models. However, the proapoptotic effects of celecoxib are not entirely understood, as normal or upregulated levels of COX-2 have been detected in various cancers after treatment (27-29). We also observed that TC-1 cells were overall slightly less sensitive to bortezomib or celecoxib at relatively low doses, and detected increased COX-2 levels after co-treatment or sequential treatment with bortezomib and celecoxib. Notably, sequential treatment with bortezomib $(10 \mathrm{nM})$ and celecoxib $(40 \mu \mathrm{M})$ effectively induced apoptosis, compared to co-treatment or sequential treatment with celecoxib and bortezomib.

The intrinsic apoptotic pathway involves dysregulation of pro- and anti-apoptotic mitochondrial proteins, and the extrinsic apoptotic pathway is triggered by the binding of tumor necrosis factor family death ligands to their appropriate death receptors (DRs) on the cell surface, followed by caspase- 8 cleavage (30). Caspase- 8 and Bid, a pro-apoptotic BH3 family member, are then cleaved by the death ligands. Expression of STAT3 is correlated with the production of high levels of anti-apoptotic gene products in cancer (31). Similarly, our results showed that sequential treatment with bortezomib and celecoxib increased the production of intrinsic apoptotic-related molecules, such as Bax and the cleaved form 
A

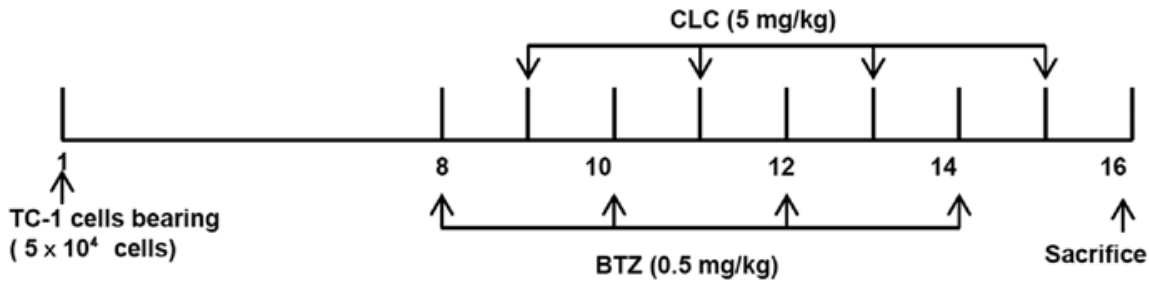

B

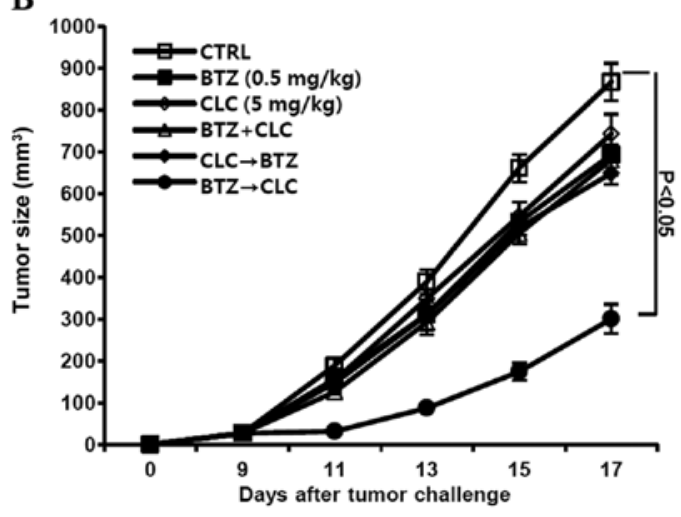

C

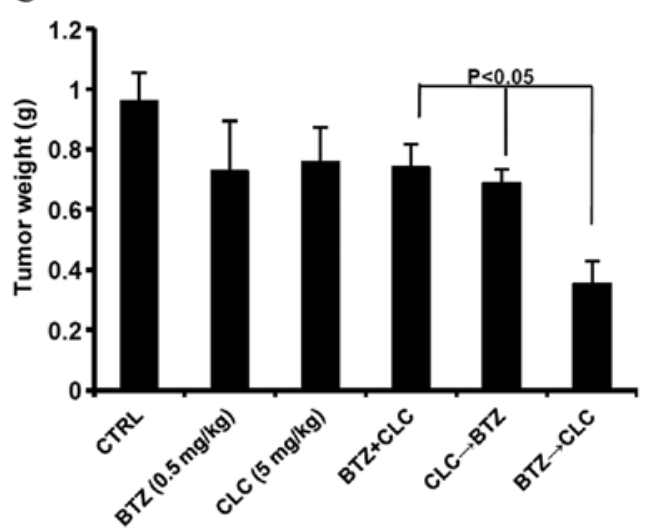

Figure 6 . Inhibition of tumor growth by sequential bortezomib and celecoxib treatment in vivo. (A) The experimental design. (B and C) C57BL/6 mice ( $=5$ per group) were challenged with TC-1 cells 1 week prior to initiating sequential bortezomib and celecoxib treatment. Each treatment group was treated via i.p. injection as indicated at day 8 after tumor challenge. Tumor size was measured at intervals of about two days. At the end of the treatment, the mice were sacrificed, and the tumors were collected and weighed $(\mathrm{P}<0.05$, difference between co-treatment or CLC $\rightarrow \mathrm{BTZ}$ and sequential BTZ $\rightarrow \mathrm{CLC}$ treatment). The data are from one representative experiment that was performed three times. BTZ, bortezomib; CLC, celecoxib.

of caspase-9, while it decreased levels of Bcl-2 and did not affect the levels of Bid and caspase-8.

STAT3 plays an important role in tumor progression by promoting cell growth and angiogenesis as well as immune evasion and inflammation. Constitutive activation of STAT3 had been reported in many cancer cell types, including cervical cancer cells (32). Activation of STAT3 has an effect on the expression of cell cycle regulator genes as well as the expression of Bcl-2 and Bax. STAT3 production also affects tumor growth through control of transient G1 to $\mathrm{S}$ phase molecules, particularly cyclin D1, which is a G0/G1 cell cycle-related protein (33). It has been reported that cyclin D1-dependent repression of STAT3 may induce apoptosis (34). Transition through G1 into $\mathrm{S}$ phase requires activation of cyclin $\mathrm{D}$ through the binding of CDK4 (4). In the next phase, cyclin E is activated by CDK2 binding (5), and cell cycle progression is regulated by CKIs such as p21. p21 in particular, when activated by p53, binds to the cyclin-CDK complex and subsequently blocks progression of the cell cycle at the G0/G1 checkpoint (35). Many studies have demonstrated that STAT3 overproduction in tumor cells is related to tumor survival (30), and decreased cyclin D1 and CDK2 levels are known to induce apoptosis in various cancer cells $(36,37)$. A previous study has shown that withaferin A, the active component of the medicinal plant Withania somnifera, significantly downregulates the expression of HPV E6 and E7 proteins in CaSki cells and restores the p53 pathway. Withaferin A also decreases the levels of STAT3 (38). Our results showed that STAT3, cyclin D1, and CDK2 levels were decreased in TC-1 cells sequentially treated with bortezomib and celecoxib. Moreover, increased levels of p21 led to cell cycle arrest, despite the lack of a concurrent upregulation in p53 expression. These results indicate that apoptosis in TC-1 cells sequentially treated with bortezomib and celecoxib may be mediated by decreased production of STAT3, cyclin D1 and CDK2 in the G1 phase even in p53-deficient cancer cells.

We detected an increase in p21 protein expression with all combined treatment regimens, which correlates with previous research indicating that $\mathrm{p} 21$ is positively regulated by CHOP under conditions of ER stress (39). The ER maintains a balance between protein synthesis and degradation (40). $\mathrm{BiP}$ is a major component of the pro-survival pathway in the unfolded protein response (UPR) and plays an important role in the ER in protein folding and assembly $(41,42)$. BiP dissociates from the three ER transmembrane receptors (PERK, ATF-6 and IRE-1 $\alpha$ ), leading to their activation and triggering the UPR. The activated PERK pathway induces downstream CHOP expression, triggering apoptosis (43). CHOP is usually produced at very low levels in the cytosol, but is produced at high levels in response to ER stress, leading to the induction of CHOP and its accumulation in the nucleus (39). p38 MAPK has been reported to promote the ER stress response (44) and cell cycle arrest in the G1 phase (45). In the present study, we observed an increased expression of CHOP and phosphorylated p38 (p-p38) after co-treatment with bortezomib and celecoxib in TC- 1 cells. Notably, our results also showed that the apoptosis of TC-1 cells sequentially treated with bortezomib and celecoxib was more closely related to cell cycle arrest and p-p38 levels than to increased CHOP expression. SB203580, an inhibitor of p-p38, affects the expression of G0/G1 cell cycle molecules, cyclin D1 and CDK2, but has no effect on CHOP. These results suggest that sequential treatment with bortezomib and celecoxib in p53-degraded cancer cells induces apoptosis through p38 MAPK-dependent cell cycle arrest rather than ER stress. 
A previous clinical trial showed that the combination of bortezomib and celecoxib can be used to treat several types of advanced solid tumors without dose-limiting toxicities, although disease progression was reported in most of the patients (46). Sequential treatment with bortezomib and celecoxib demonstrated the most effective antitumor effects in vivo study. Unfortunately, tumor growth was not completely inhibited by sequential treatment with bortezomib and celecoxib. Based on these results, further studies are needed to clarify related apoptotic pathways and new target molecules for the development of a combined anticancer chemotherapy.

The present study supports the concept that apoptosis induced by ER stress is enhanced by treatment with a combination of bortezomib and celecoxib. In addition, our results suggest that the sequential administration of bortezomib and celecoxib may be an effective strategy for inducing apoptosis in p53-degraded cancer cells via p38-mediated cell cycle regulation.

\section{Acknowledgements}

The present study was supported by the Basic Science Research Program through the National Research Foundation of Korea (NRF) funded by the Ministry of Education, Science and Technology (NRF-2013-R1A1A2-010668, NRF-2012R1A1A3-013468).

\section{References}

1. Jemal A, Siegel R, Ward E, Hao Y, Xu J, Murray T and Thun MJ: Cancer statistics, 2008. CA Cancer J Clin 58: 71-96, 2008.

2. ZurHausen $\mathrm{H}$ and de Villiers EM: Human papillomaviruses. Annu Rev Microbiol 48: 27-47, 1994.

3. Maher DM, Bell MC, O'Donnell EA, Gupta BK, Jaggi M and Chauhan SC: Curcumin suppresses human papillomavirus oncoproteins, restores $\mathrm{p} 53, \mathrm{Rb}$, and PTPN13 proteins and inhibits benzo[a]pyrene-induced upregulation of HPV E7. Mol Carcinog 50: 47-57, 2011.

4. Werness BA, Levine AJ and Howley PM: Association of human papillomavirus types 16 and 18 E6 proteins with $\mathrm{p} 53$. Science 248: 76-79, 1990.

5. Alani RM and Munger K: Human papillomaviruses and associated malignancies. J Clin Oncol 16: 330-337, 1998.

6. Ortega S, Malumbres M and Barbacid M: Cyclin D-dependent kinases, INK4 inhibitors and cancer. Biochim Biophys Acta 1602: 73-87, 2002.

7. Sherr CJ and Roberts JM: Living with or without cyclins and cyclin-dependent kinases. Genes Dev 18: 2699-2711, 2004.

8. Vogelstein B, Lane D and Levine AJ: Surfing the p53 network. Nature 408: 307-310, 2000.

9. Bahnassy AA, Zekri AR, Alam El-Din HM, Aboubakr AA, Kamel K, El-Sabah MT and Mokhtar NM: The role of cyclins and cyclin inhibitors in the multistep process of HPV-associated cervical carcinoma. J Egypt Natl Canc Inst 18: 292-302, 2006.

10. Lin KY, Guarnieri FG, Staveley-O'Carroll KF, Levitsky HI, August JT, Pardoll DM and Wu TC: Treatment of established tumors with a novel vaccine that enhances major histocompatibility class II presentation of tumor antigen. Cancer Res 56: 21-26, 1996.

11. Tseng CW, Monie A, Wu CY, Huang B, Wang MC, Hung CF and Wu TC: Treatment with proteasome inhibitor bortezomib enhances antigen-specific CD8 ${ }^{+} \mathrm{T}$-cell-mediated antitumor immunity induced by DNA vaccination. J Mol Med 86: 899-908, 2008.

12. Malara N, Focà D, Casadonte F, Sesto MF, Macrina L, Santoro L Scaramuzzino M, Terracciano R and Savino R: Simultaneous inhibition of the constitutively activated nuclear factor kappaB and of the interleukin- 6 pathways is necessary and sufficient to completely overcome apoptosis resistance of human U266 myeloma cells. Cell Cycle 7: 3235-3245, 2008.
13. Wen J, Feng Y, Huang W, Chen H, Liao B, Rice L Preti HA, Kamble RT, Zu Y, Ballon DJ and Chang CC: Enhanced antimyeloma cytotoxicity by the combination of arsenic trioxide and bortezomib is further potentiated by p38 MAPK inhibition. Leuk Res 34: 85-92, 2010.

14. Eberhart CE, Coffey RJ, Radhika A, Giardiello FM, Ferrenbach S and DuBois RN: Upregulation of cyclooxygenase-2 gene expression in human colorectal adenomas and adenocarcinomas. Gastroenterology 107: 1183-1188, 1994.

15. Kulkarni S, Rader JS, Zhang F, Liapis H, Koki AT, Masferrer JL, Subbaramaiah K and Dannenberg AJ: Cyclooxygenase-2 is overexpressed in human cervical cancer. Clin Cancer Res 7: 429-434, 2011.

16. Kim K, Jeon YT, Park IA, Kim JW, Park NH, Kang SB, Lee HP, and Song YS: Cyclooxygenase-2 expression in cervical intraepithelial neoplasia. Ann NY Acad Sci 1171: 111-115, 2009.

17. Subbaramaiah K and Dannenberg AJ: Cyclooxygenase-2 transcription is regulated by human papillomavirus 16 E6 and E7 oncoproteins: evidence of a corepressor/coactivator exchange. Cancer Res 67: 3976-3985, 2007.

18. Ferrandina G, Ranelletti FO, Legge F, Lauriola L, Salutari V, Gessi M, Testa AC, Werner U, Navarra P, Tringali G, Battaglia A and Scambia G: Celecoxib modulates the expression of cyclooxygenase-2, ki67, apoptosis-related marker, and microvessel density in human cervical cancer: a pilot study. Clin Cancer Res 9: 4324-4331, 2003.

19. Sareddy GR, Geeviman K, Ramulu C and Babu PP: The nonsteroidal anti-inflammatory drug celecoxib suppresses the growth and induces apoptosis of human glioblastoma cells via the NF-кB pathway. J Neurooncol 106: 99-109, 2011.

20. Kardosh A, Golden EB, Pyrko P, Uddin J, Hofman FM, Chen TC Louie SG, Petasis NA and Schönthal AH: Aggravated endoplasmic reticulum stress as a basis for enhanced glioblastoma cell killing by bortezomib in combination with celecoxib or its non-coxib analogue, 2,5-dimethyl-celecoxib. Cancer Res 68: 843-851, 2008.

21. Mukhopadhyay I, Sausville EA, Doroshow JH and Roy KK: Molecular mechanism of adaphostin-mediated G1 arrest in prostate cancer (PC-3) cells: signaling events mediated by hepatocyte growth factor receptor, c-Met, and p38 MAPK pathways. J Biol Chem 281: 37330-37344, 2006.

22. Fujita T, Doihara H, Washio K, Ino H, Murakami M, Naito M and Shimizu N: Antitumor effects and drug interactions of the proteasome inhibitor bortezomib (PS341) in gastric cancer cells. Anticancer Drugs 18: 677-686, 2007.

23. Zhang X, Morham SG, Langenbach R and Young DA: Malignant transformation and antineoplastic actions of nonsteroidal antiinflammatory drugs (NSAIDs) on cyclooxygenase-null embryo fibroblasts. J Exp Med 190: 451-459, 1999.

24. Nawrocki ST, Carew JS, Pino MS, Highshaw RA, Dunner K Jr, Huang P, Abbruzzese JL and McConkey DJ: Bortezomib sensitizes pancreatic cancer cells to endoplasmic reticulum stress-mediated apoptosis. Cancer Res 65: 11658-11666, 2005.

25. Obeng EA, Carlson LM, Gutman DM, Harrington WJ Jr, Lee KP and Boise LH: Proteasome inhibitors induce a terminal unfolded protein response in multiple myeloma cells. Blood 107: 4907-4916, 2006.

26. Fribley A and Wang CY: Proteasome inhibitor induces apoptosis through induction of endoplasmic reticulum stress. Cancer Biol Ther 5: 745-748, 2006.

27. Hanif R, Pittas A, Feng Y, Koutsos MI, Qiao L, Staiano-Coico, L Shiff SI and Rigas B: Effects of nonsteroidal anti-inflammatory drugs on proliferation and on induction of apoptosis in colon cancer cells by a prostaglandin-independent pathway. Biochem Pharmacol 52: 237-245, 1996.

28. Kardosh A, Blumenthal M, Wang WJ, Chen TC and Schönthal AH: Differential effects of selective COX-2 inhibitors on cell cycle regulation and proliferation of glioblastoma cell lines. Cancer Biol Ther 3: 9-16, 2004.

29. Arico S, Pattingre S, Bauvy C, Gane P, Barbat A, Codogno P and Ogier-Denis E: Celecoxib induces apoptosis by inhibiting 3 -phosphoinositide-dependent protein kinase-1 activity in the human colon cancer HT-29 cell line. J Biol Chem 277: 27613-27621, 2002.

30. Elmore S: Apoptosis: a review of programmed cell death. Toxicol Pathol 35: 495-516, 2007.

31. Kawano M, Hirano T, Matsuda T, Taga T, Horii Y, Iwato K, Asaoku H, Tang B, Tanabe O, Tanaka H, Kuramoto A and Kishimoto T: Autocrine generation and requirement of BSF-2/ IL-6 for human multiple myelomas. Nature 332: 83-85, 1988. 
32. Chen J, Wang J, Lin L, He L, Wu Y, Zhang L, Yi Z, Chen Y, Pang $X$ and Liu M: Inhibition of STAT3 signaling pathway by nitidine chloride suppressed the angiogenesis and growth of human gastric cancer. Mol Cancer Ther 11: 277-287, 2011.

33. Wang J, Li X, Lu X and Pi L: The regulation of stat 3 signal transduction pathway to G1 to $\mathrm{S}$ phase of laryngocarcinoma cell. Lin Chung Er Bi Yan Hou Tou Jing Wai Ke Za Zhi 22: 699-703, 2008 (In Chinese).

34. Ishii Y, Pirkmaier A, Alvarez JV, Frank DA, Keselman I, Logothetis D, Mandeli J, O'Connell MJ, Waxman S and Germain D: Cyclin D1 overexpression and response to bortezomib treatment in a breast cancer model. J Natl Cancer Inst 98: 1238-1247, 2006.

35. Harper JV and Brooks G: The mammalian cell cycle: an overview. Methods Mol Biol 296: 113-153, 2005.

36. Wang J, Wang Q, Cui Y, Liu ZY, Zhao W, Wang CL, Dong Y, Hou L, Hu G, Luo C, Chen J and Lu Y: Knockdown of cyclin D1 inhibits proliferation, induces apoptosis, and attenuates the invasive capacity of human glioblastoma cells. J Neurooncol 106: 473-484, 2011

37. Shapiro GI: Cyclin-dependent kinase pathways as targets for cancer treatment. J Clin Oncol 24: 1770-1783, 2006

38. Munagala R, Kausar H, Munjal C and Gupta RC: Withaferin A induces p53-dependent apoptosis by repression of HPV oncogenes and upregulation of tumor suppressor proteins in human cervical cancer cells. Carcinogenesis 32: 1697-1705, 2011

39. Mihailidou C, Papazian I, Papavassiliou AG and Kiaris H: CHOP-dependent regulation of p21/waf1 during ER stress. Cell Physiol Biochem 25: 761-766, 2010.
40. Mori T, Hayashi T and Su TP: Compromising sigma-1 receptors at the ER renders cytotoxicity to physiologicallyrelevant concentrations of dopamine in a NF- $\kappa \mathrm{B} / \mathrm{Bcl}-2$-dependent mechanism: Potential relevance to Parkinson's disease. J Pharmacol Exp Ther 341: 663-671, 2012.

41. Oyadomari S and Mori M: Roles of CHOP/GADD153 in endoplasmic reticulum stress. Cell Death Differ 11: 381-389, 2004.

42. Shimazawa M, Miwa A, Ito $Y$, Tsuruma K, Aihara M and Hara H: Involvement of endoplasmic reticulum stress in optic nerve degeneration following $N$-methyl-D-aspartate-induced retinal damage in mice. J Neurosci Res 90: 1960-1969, 2012.

43. Huang KH, Kuo KL, Chen SC, Weng TI, Chuang YT, Tsai YC, $\mathrm{Pu}$ YS, Chiang CK and Liu SH: Down-regulation of glucoseregulated protein (GRP) 78 potentiates cytotoxic effect of celecoxib in human urothelial carcinoma cells. PLoS One 7: e33615, 2012.

44. Shimada Y, Kobayashi H, Kawagoe S, Aoki K, Kaneshiro E, Shimizu H, Eto Y, Ida $\mathrm{H}$ and Ohashi T: Endoplasmic reticulum stress induces autophagy through activation of p38 MAPK in fibroblasts from Pompe disease patients carrying c.546G $>\mathrm{T}$ mutation. Mol Genet Metab 104: 566-573, 2011.

45. Lee B, Kim CH and Moon SK: Honokiol causes the p21WAF1mediated G(1)-phase arrest of the cell cycle through inducing p38 mitogen activated protein kinase in vascular smooth muscle cells. FEBS Lett 580: 5177-5184, 2006.

46. Hayslip J, Chaudhary U, Green M, Meyer M, Dunder S, Sherman C, Salzer S, Kraft A and Montero AJ: Bortezomib in combination with celecoxib in patients with advanced solid tumors: a phase I trial. BMC Cancer 7: 221, 2007. 